\title{
Rifampicin-Induced Nephrotoxicity in a Tuberculosis Patient: Treatment Dilemma?
}

\author{
António Grilo Novais ${ }^{1}$, Cláudio Silva², Ana Rita Coelho ${ }^{3}$, Roberto Silva ${ }^{3}$, Ana Cláudia Carvalho ${ }^{4}$ \\ ${ }^{1}$ Internal Medicine Department, Centro Hospitalar Tondela-Viseu, E.P.E, Portugal \\ ${ }^{2}$ Infectious Diseases Department, Centro Hospitalar Universitário São João, Porto, Portugal \\ ${ }^{3}$ Pathology Department, Centro Hospitalar Universitário São João, Porto, Portugal \\ ${ }^{4}$ Infectious Diseases Department, Hospital de Braga, Braga, Portugal
}

Received: 15/08/2021

Accepted: 06/09/2021

Published: $14 / 10 / 2021$

How to cite this article: Grilo Novais A, Silva C, Coelho AR, Silva R, Calvalho AC. Rifampicin-induced nephrotoxicity in a tubercolosis patient: treatment dilemma? EJCRIM 2021;8: doi:10.12890/2021_002833.

Conflicts of Interests: The authors declare there are no competing interests.

This article is licensed under a Commons Attribution Non-Commercial 4.0 License

\section{ABSTRACT}

Acute kidney injury related to rifampin is usually a clinical diagnosis. We report a case of a man being treated for pulmonary tuberculosis with acute tubulointerstitial nephritis related to rifampicin.

\section{LEARNING POINTS}

- Rifampicin can cause acute kidney injury.

- Renal impairment may develop weeks after rifampicin administration was started.

\section{KEYWORDS}

Acute tubulointerstitial nephritis, rifampicin-induced, tuberculosis

\section{INTRODUCTION}

Rifampicin is one of the most effective and widely used antibiotics in tuberculosis (TB) treatment ${ }^{[1]}$. Adverse effects, such as acute renal failure, are rarely described in the literature and most patients recover complete renal function upon discontinuation of therapy ${ }^{[2]}$. The following case report describes a 43-year-old male presenting with pulmonary TB who developed rifampicin-induced renal toxicity late during treatment. Renal function was fully recovered upon discontinuation of the medication and treatment with corticosteroids but the best approach is as yet undefined.

\section{CASE DESCRIPTION}

A 43-year-old male construction worker was admitted to the emergency room with an 8-week history of cough, fever, night sweats and weight loss. He was a smoker (smoking load 22 pack-years) but otherwise healthy. Chest radiography disclosed interstitial opacities located to the right lung, mainly in the upper lobe (Fig. 1), and he was admitted to the infectious diseases ward with the suspicion of pulmonary TB. HIV screening was negative. Direct examination of sputum stained positive for acid-fast bacilli and treatment with isoniazid, rifampicin, pyrazinamide and ethambutol daily was initiated. Five days later, he developed chest pain, without other symptoms suggestive of heart failure, and the findings of coronary angiography with left ventriculography were compatible with Takotsubo syndrome with depressed left ventricular systolic function. As per treatment recommendations, a beta blocker and aspirin were initiated and the patient recovered. 


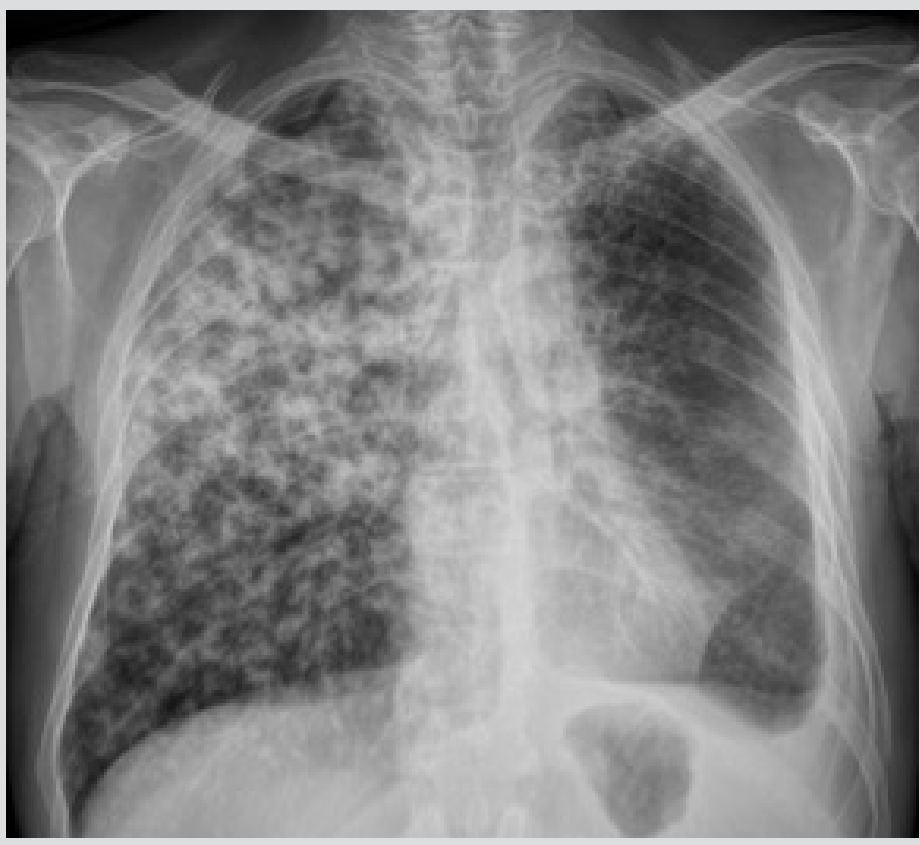

Figure 1. Chest radiography disclosed interstitial opacities located to the right lung,

On the 60th day of inpatient stay, asymptomatic acute kidney injury (AKI) was documented, with maximum creatinine levels of 1.8 mg/dl, diuresis preserved. Twenty-four-hour urine revealed a urinary creatinine clearance of $20 \mathrm{ml} / \mathrm{min} / 1.73 \mathrm{~m}^{2}$ and subnephrotic proteinuria of $2.8 \mathrm{~g}$. He had glycosuria but no haematuria. Urine sediment had no eosinophils, was crystal-free and contained a very low concentration of proteins. The blood eosinophil percentage was normal (7\%). The kidneys were of normal size and structure on the abdominal ultrasound. Autoimmune screening was negative.

AA amyloidosis due to pulmonary TB vs acute interstitial nephritis due to rifampicin were the main differentials as to the aetiology of renal toxicity. Renal biopsy was performed, revealing interstitial inflammatory infiltrates of lymphocytes and eosinophils, acute tubular injury (Fig. 2) and tubulitis with infiltrating mononuclear inflammatory cells (Fig. 3), thereby confirming the diagnosis of acute interstitial nephritis. No amyloid deposits were found (Congo red).

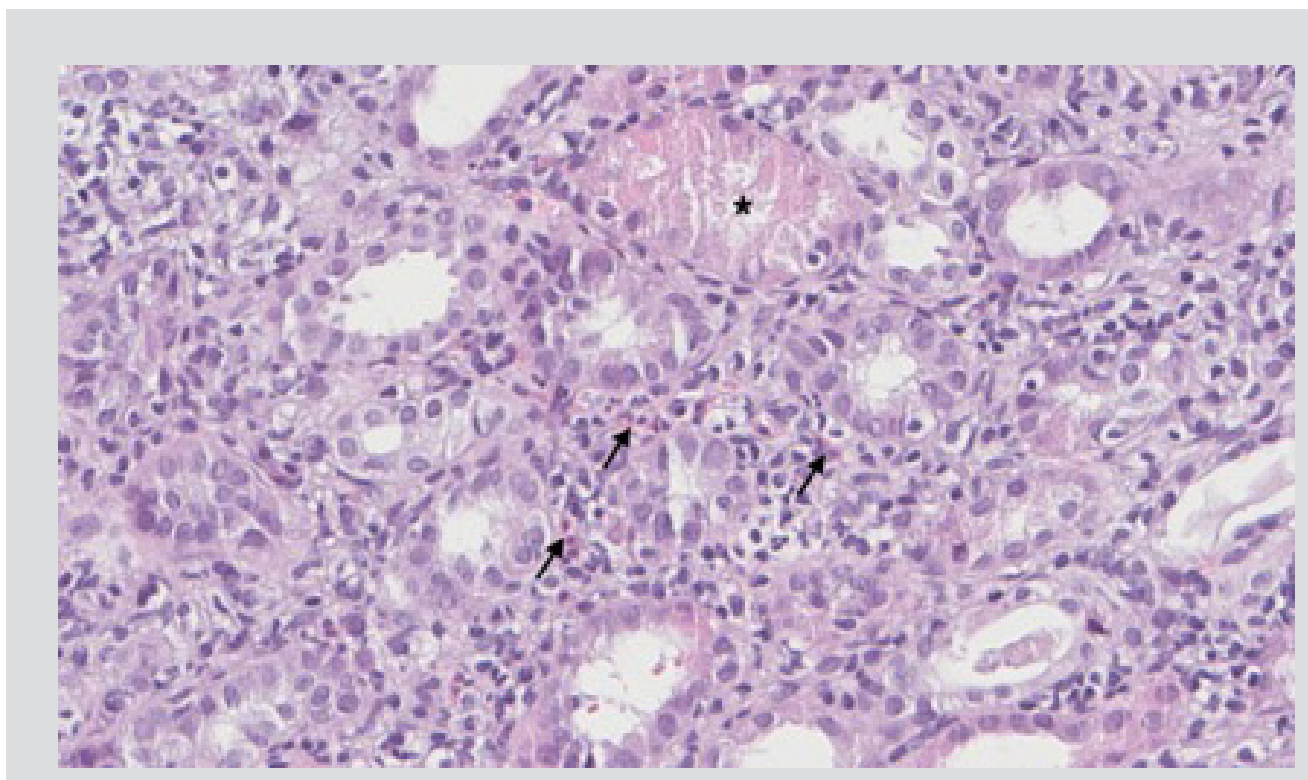

Figure 2. Drug-induced acute interstitial nephritis (HE, 400x): interstitial inflammatory infiltrate of lymphocytes and eosinophils (arrows) and acute tubular injury (asterisk) 


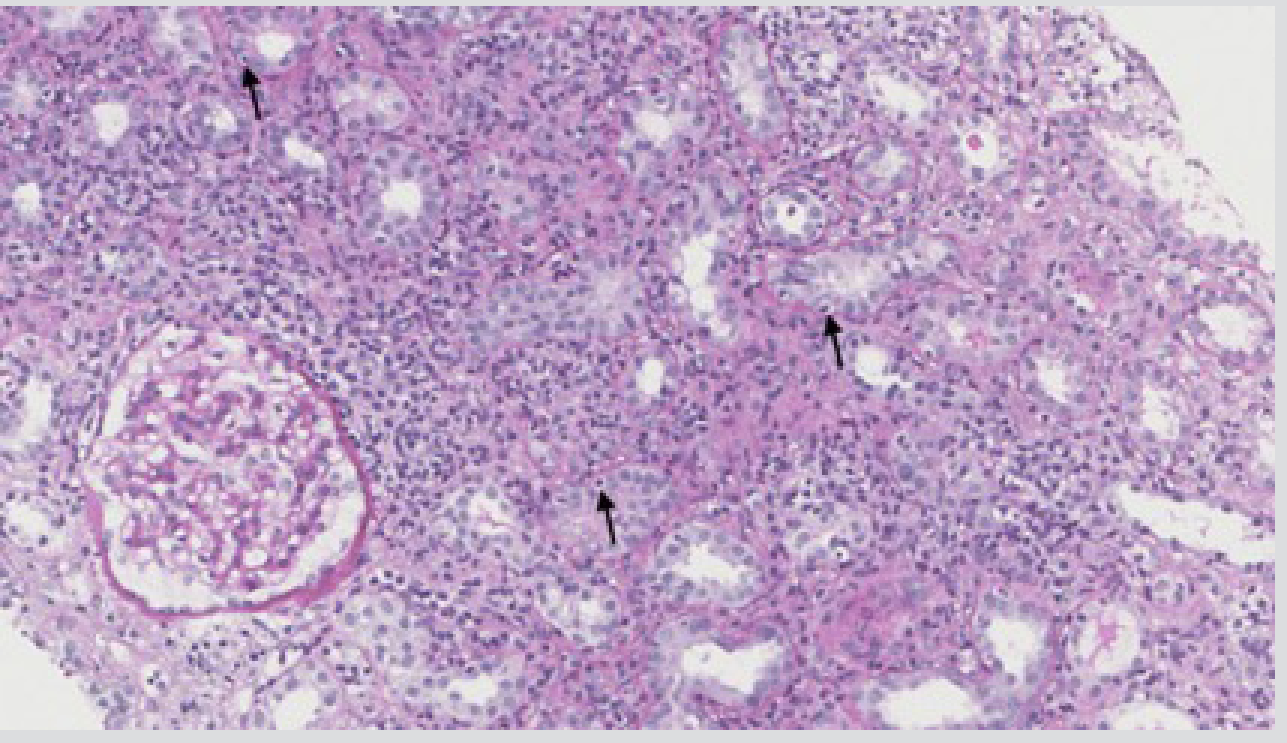

Figure 3. Drug-induced acute interstitial nephritis (PAS, 200x): tubulitis with infiltrating mononuclear inflammatory cells (arrows)

Rifampicin was discontinued and replaced with levofloxacin. Nonetheless, renal function did not improve over the subsequent week and after a multidisciplinary ward meeting (internal medicine, infectious diseases and nephrology) it was decided to add prednisolone $1 \mathrm{mg} / \mathrm{kg} /$ day, prescribed for 14 days (with progressive dose reduction afterwards), to the treatment regimen. The patient recovered complete renal function.

\section{DISCUSSION}

Renal biopsy is the "gold standard" for the diagnosis of interstitial nephritis ${ }^{[3]}$. A high rate of suspicion of AKI associated with rifampicin should be considered in patients undergoing TB therapy who develop progressive deterioration of renal function. Although rare, rifampicin is the most likely anti-TB drug to be associated with renal damage ${ }^{[1,2]}$. Early diagnosis and discontinuation of the offending drug are extremely important for renal function recovery. The use of corticosteroids is controversial and more studies are needed to demonstrate the clinical benefit of using such a treatment strategy ${ }^{[3,4]}$.

In conclusion, this clinical case highlights rifampicin as a possible cause of AKI. It is important to stress this atypical presentation of AKI: causes may be multifactorial. Careful evaluation and long-term surveillance of these patients remains necessary ${ }^{[5]}$.

\section{REFERENCES}

1. Manika K, Tasiopoulou K, Vlogiaris L, Lada M, Papaemmanouil S, Zarogoulidis K, et al. Rifampicin-associated acute renal failure and hemolysis: a rather uncommon but severe complication. Ren Fail 2013;35(8):1179-1181.

2. Beebe A, Seaworth B, Patil N. Rifampicin-induced nephrotoxicity in a tuberculosis patient. J Clin Tuberc Other Mycobact Dis 2015;1:13-15.

3. Joyce E, Glasner P, Ranganathan S, Swiatecka-Urban A. Tubulointerstitial nephritis: diagnosis, treatment, and monitoring. Pediatr Nephrol $2017 ; 32(4): 577-587$.

4. Clarkson MR, Giblin L, O'Connell FP, O'Kelly P, Walshe JJ, Conlon P, et al. Acute interstitial nephritis: clinical features and response to corticosteroid therapy. Nephrol Dial Transplant 2004;19(11):2778-2783.

5. González E, Gutiérrez E, Galeano C, Chevia C, de Sequera P, Bernis C, et al. Early steroid treatment improves the recovery of renal function in patients with drug-induced acute interstitial nephritis. Kidney Int 2008;73(8):940-946. 